\title{
Laser linear encoder with both high fabrication and head-to-scale tolerances
}

\author{
Chyan-Chyi Wu, ${ }^{1}$ Wen-Jong Wu, ${ }^{2}$ Zheng-Seng Pan, ${ }^{3}$ and Chih-Kung Lee ${ }^{2,4, *}$ \\ ${ }^{1}$ Center for Measurement Standards, Industrial Technology Research Institute, 321 Kuang-Fu Road, Hsinchu 300, Taiwan \\ ${ }^{2}$ Department of Engineering Science and Ocean Engineering, National Taiwan University, Taipei 10617, Taiwan \\ ${ }^{3}$ Chi Mei Optoelectronics, Huanshi Road, Southern Taiwan Science Park, Sinshih Township, \\ Tainan County 74147 , Taiwan \\ ${ }^{4}$ Institute of Applied Mechanics, National Taiwan University, No. 1 Roosevelt Road, Section 4, Taipei 10617, Taiwan \\ *Corresponding author: cklee@mems.iam.ntu.edu.tw
}

Received 26 January 2006; revised 12 December 2006; accepted 7 March 2007;

posted 14 March 2007 (Doc. ID 67502); published 15 May 2007

\begin{abstract}
We introduce a new configuration for the optical head of a newly developed diffractive laser encoder system. This configuration has a high manufacturing tolerance and a high head-to-scale alignment tolerance, both of which can enhance the wider potential applicability of this newly designed laser encoder. The measurement principles of the encoder are discussed and detailed. We optimized the grating shape and analyzed the impact of the optical components and their arrangement on the measurement error. The head-to-scale alignment tolerance and the arrangement of components in the encoder were also determined. Finally, the measurement performance was evaluated and analyzed. Under nonenvironmentally controlled conditions, the measurement accuracy was found to be $37.3 \mathrm{~nm}$ with a standard deviation of $25.4 \mathrm{~nm}$. (C) 2007 Optical Society of America
\end{abstract}

OCIS codes: $\quad 050.1950,230.2090,120.0120,230.0230$.

\section{Introduction}

A laser encoder is an optical interferometer that uses a grating to generate interference signals proportional to the grating displacement. By using a grating as the measurement scale, the laser encoder is less prone to influences by environmental disturbances when compared with traditional laser interferometers [1]. However, traditional laser encoders possess optical configurations that are difficult to manufacture and usually have a tight head-to-scale tolerance that can restrict its applicability for many applications [2-5]. We describe our newly developed encoder that we call the DiLENS (an acronym for diffractive laser encoder system). Our DiLENS integrates the advantages of a Michelson interferometer, homodyne interferometry (whose phase relates to displacement in trigonometry means, i.e., nonlinear), a quadrature detection feature, and wavefront conjugate optics into one system. Our newly developed system is capable of

0003-6935/07/163169-08\$15.00/0

(C) 2007 Optical Society of America achieving measurement resolutions in nanometers and total measurement ranging in centimeters, all the while exhibiting a high environmental tolerance.

To reduce the dimensions of our DiLENS, a He-Ne gas laser was replaced with a semiconductor laser. With this reduced size, the system was less affected by external environmental noise such as heat and air turbulence. Substitution of the He-Ne laser by a semiconductor laser was not totally advantageous because we then had to contend with undesirable noise such as mode hopping, as well as additional errors from misalignment of the optical components.

We describe the configuration and principles of this innovative encoder as well as the head-to-scale alignment and manufacturing tolerances of this configuration based on our experimental data and results.

\section{Optomechanical Configuration}

Figure 1 shows the schematic optomechanical configuration of the optical head. One can see that orthogonal or parallel optical paths are present in all the encoder components except for the focusing lens, 


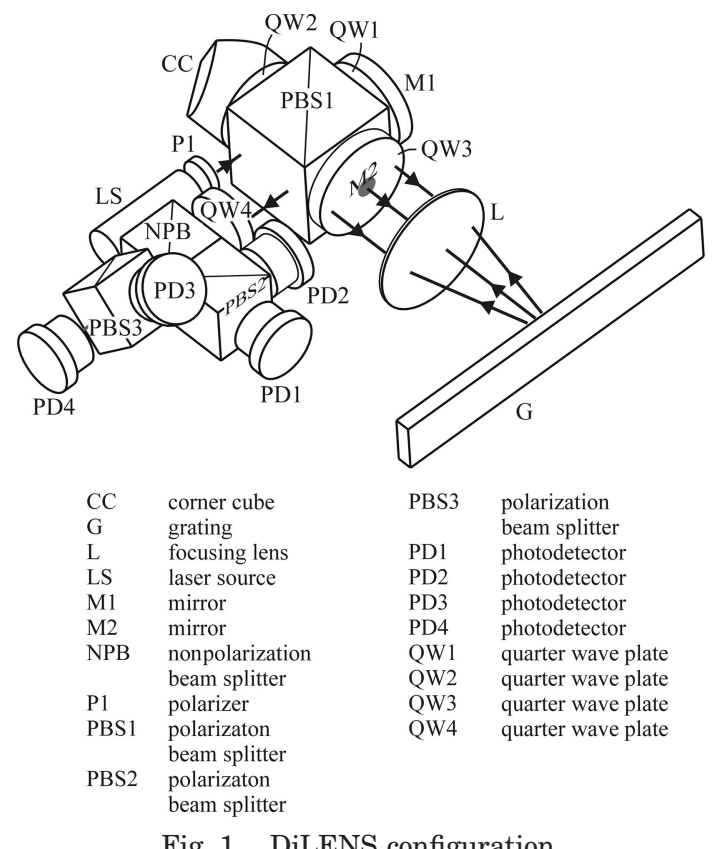

Fig. 1. DiLENS configuration.

which can thus simplify mechanical assembly and manufacturing. The optomechanical configuration of the optical head consists of three parts: (1) a wavefront conjugate optics formed by use of a combination of a focal lens and a grating, (2) an optimized grating scale, and (3) a circular polarization interferometer.

The emitted laser beam was split into two beams after it passed through the polarization beam splitter (PBS1) (the $s$ and $p$ polarization states are shown in Fig. 2). The $s$ polarization becomes left circularly polarized after reflection by the polarization split film of PBS1 and after going through quarter-wave plate 2 (QW2). This circularly polarized beam then passes

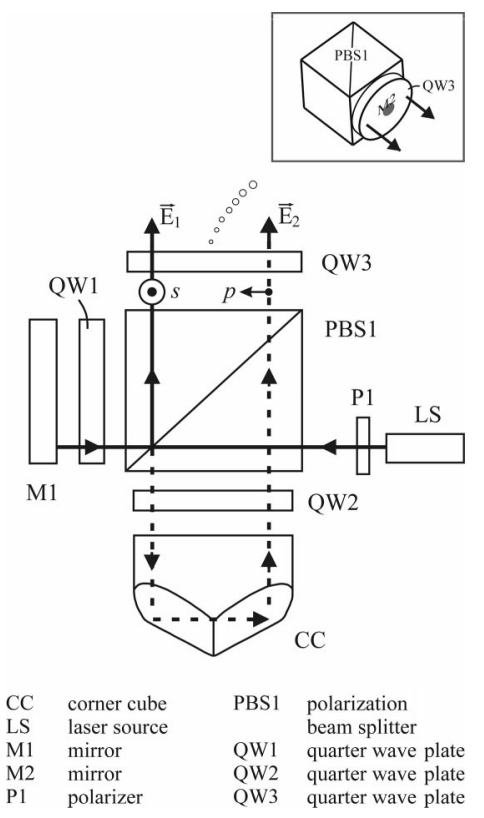

Fig. 2. Schematic of the splitter system. into the corner cube (CC). After three full reflections, light passes through the QW2 and turns into a $p$-polarized beam. This $p$-polarized beam then goes through the polarization split film of PBS1 along the direction that is perpendicular to the incident beam (Fig. 2). After it passes through the quarterwaveplate (QW3), it then turns into circular polarization. It then enters the linear grating scale $\mathrm{G}$ after going through focal lens L. The [-1] order diffractive laser diffracted by grating scale $\mathrm{G}$ goes through lens $\mathrm{L}$ and is then reflected by mirror 2 (M2). The reflected circularly polarized beam goes through lens L again and is diffracted by grating scale G. The generated $[+1]$ order diffractive laser goes along the original optical path and turns into $s$ polarization after going through QW3. It then goes back to PBS1 and is reflected by the polarization split film. After going through QW1, it is then reflected by $\mathrm{M} 1$ and goes though QW1 again, where the laser becomes $p$ polarized and then goes through PBS1. This $p$-polarized beam then enters a circular polarization interferometer section and goes through QW4 (Fig. 1). A right circularly polarized (rcp) light beam $\mathbf{E}_{\text {rcp }}$ can then be produced.

Furthermore, the path for the $p$-polarized beam, after it has been split by PBS1, goes through QW1 and becomes right circularly polarized. This circularly polarized beam is then reflected by M1, where it goes though QW1 again and becomes $s$ polarized. The $s$-polarized beam is then reflected by the polarization split film of PBS1 where it goes through QW3 and becomes left circularly polarized. It then goes through lens L and enters grating scale G. The [+1] order diffractive laser is diffracted by grating scale $\mathrm{G}$ and goes through lens $\mathrm{L}$. The laser is then reflected by $\mathrm{M} 2$, which is a silver thin film coated portion of QW3. The reflected left circularly polarized beam goes through lens $\mathrm{L}$ again and is diffracted by the grating scale. The generated $[-1]$ order diffractive laser goes back to PBS1 along the original path. It goes through the polarization split film and QW2. After reflection by the $\mathrm{CC}$ and after going through the QW2 once more, the laser beam becomes $s$ polarized and is then reflected by the polarization split film of PBS1. This $s$ polarization enters the circular polarization interferometer and produces the left circularly polarized (lcp) beam $\mathbf{E}_{\text {lcp }}$ after it goes through QW4 (Fig. 1).

\section{Measurement Principles}

Both circularly polarized light beams, $\mathbf{E}_{\mathrm{rcp}}$ and $\mathbf{E}_{\mathrm{lcp}}$, experience two Doppler frequency shifts. Frequency shifts $\Delta \omega^{+1}$ and $\Delta \omega^{-1}$ are represented as

$$
\begin{aligned}
& \Delta \omega^{+1}=2 \Delta \omega=(4 \pi / \lambda) u_{X} \sin \theta, \\
& \Delta \omega^{-1}=-2 \Delta \omega=-(4 \pi / \lambda) u_{X} \sin \theta .
\end{aligned}
$$

As shown in Fig. 3, $u_{X}$ is the moving speed of the grating scale along the $X$ axis, and $\theta$ is the angle between the incident direction of the laser from the optical head to the grating scale and the outer normal 


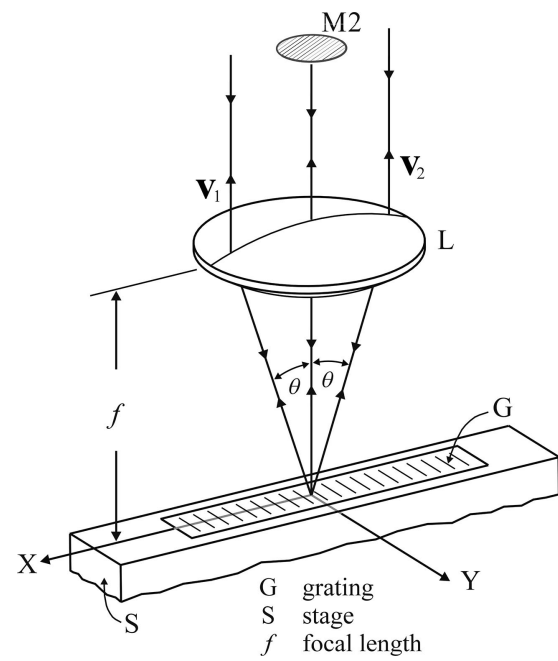

Fig. 3. Wave propagation near grating G; First diffraction path: lens $\mathrm{L} \rightarrow$ inclined incidence to grating $\mathrm{G} \rightarrow$ lens $\mathrm{L} \rightarrow$ mirror $\mathrm{M} 2$. Second diffraction path: mirror $\mathrm{M} 2 \rightarrow$ lens $\mathrm{L} \rightarrow$ grating $\mathrm{G} \rightarrow$ lens $\mathrm{L}$; the diffracted electric field along left path $\mathbf{V}_{1}$ and the diffracted electric field along right path $\mathbf{V}_{2}$. (Note: $\theta$ is the angle of incidence for the first diffraction and is also the angle of diffraction for the second diffraction.)

direction of the grating scale. In addition, $\theta$ can also be the angle between the direction of the diffractive laser beam and the normal to the grating scale. Consider the case when the grating scale moves along the $X$ axis at a speed of $u_{X}$ in small time interval $\mathrm{d} t$. The corresponding phase change $\mathrm{d} \Phi$ of the orthogonal signal can be written as

$$
\mathrm{d} \Phi=(8 \pi / \lambda) u_{X} \mathrm{~d} t \sin \theta .
$$

Thus the total phase change during the time from 0 to $t$ can be obtained by integration of Eq. (3). Displacement of grating scale $\Delta X$ and phase change $\Phi$ of the orthogonal signal can be written as

$$
\Phi=(8 \pi / \lambda) \Delta X \sin \theta .
$$

Furthermore, for the design of the optical heads shown in Fig. 3, the relationship $\sin \theta=\lambda / d$ applies [6], where $d$ is the grating pitch. Therefore, the relationship between displacement $\Delta X$ of the grating scale (displacement along the $X$ axis) and phase change $\Phi$ of the orthogonal signal can be shown to be

$$
\Delta X=(\Phi d / 8 \pi)
$$

Equation (5) implies that the grating scale motion along the $X$ axis for one grating pitch produces four interference signal periods.

\section{Grating Scale Geometric Optimization}

Figure 3 shows the wave propagation near grating G. After the second diffraction, electric fields $\mathbf{V}_{1}$ and $\mathbf{V}_{2}$ of the left and right paths can be written, respectively, as

$$
\begin{aligned}
& \mathbf{V}_{1}=\left\{\begin{array}{c}
k_{p}(\theta) k_{p}(0) \\
k_{s}(\theta) k_{s}(0) i
\end{array}\right\}, \\
& \mathbf{V}_{2}=\left\{\begin{array}{c}
k_{p}(\theta) k_{p}(0) \\
-k_{s}(\theta) k_{s}(0) i
\end{array}\right\},
\end{aligned}
$$

where $k_{p}(\theta), k_{s}(\theta), k_{p}(0)$, and $k_{s}(0)$ are the first-order diffraction efficiencies of the $p$ - and $s$-polarized light that impinges onto the grating scale at an angle of $\theta$ or 0 with respect to the grating normal; see Fig. 3 . For the optical arrangement in Fig. 1, four photodetectors, PD1-PD4, were collected in the second diffraction \pm 1 order beams. The signals detected from PD2, PD3, and PD4 had phase differences, respectively, of $\pi, \pi / 2$, and $3 \pi / 2$ with respect to PD1. Using a differential amplification technique, the direct current (DC) term of PD1 and PD2 can be removed; this technique can also apply to PD3 and PD4. In the end, we obtained two signals, $I_{1} \propto$ $4 A^{2} \cos (4 \Delta \omega t)$ and $I_{2} \propto 4 A^{2} \sin (4 \Delta \omega t)$, where $A=$ $\left[k_{p}(\theta) k_{p}(0)+k_{s}(\theta) k_{s}(0)\right] / \sqrt{2}$. It is clear that $I_{1}$ and $I_{2}$ comprise the Lissajous pattern for phase decoding. Generally, light beams with $p$ and $s$ polarizations undergo different diffraction efficiencies from a grating. However, in our case, $I_{1}$ and $I_{2}$ demonstrate that the signals of the DiLENS are not affected by the different diffraction efficiencies for $p$ - and $s$-polarized input light beams.

To improve the manufacturing tolerance and the strength of the interference light beams of the encoder, grating scales with different surface characteristics were analyzed using GSolver software. In other words, $\left[k_{p}(\theta) k_{p}(0)+k_{s}(\theta) k_{s}(0)\right]^{2}$ was used as the metric to optimize the grating scale design [7]. The gratings analyzed here include square wave gratings, delta wave gratings and sine wave gratings. The results show that the most effective grating shape for the diffractive laser encoder was a sine wave phase grating with a $1.6 \mu \mathrm{m}$ grating pitch. The optimal grating depth obtained was $160 \mathrm{~nm}$; see Fig. 4. Compared with the grating scale of commercial laser encoders

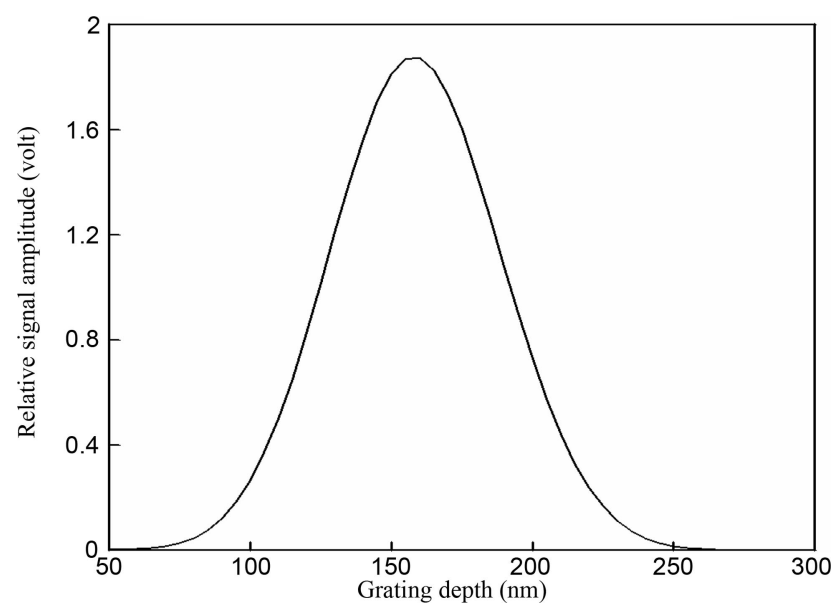

Fig. 4. Optimal grating depth $(160 \mathrm{~nm})$ for the DiLENS as calculated with the GSolver. 
of the same level (a sinusoidal phase grating with $90 \mathrm{~nm}$ depth), the optimized grating scale enhances the beam strength by at least six times [8].

\section{Optical Analysis}

The coordinate system used for the optical analysis can be seen in Fig. 5. In an ideal case, the electric fields that enter the photodetectors can be expressed in an $x-y$ coordinate, such as $\left(\cos \alpha \sin \beta_{3} \cos (\omega t+\varphi)\right.$, $\cos \alpha \cos \beta_{3} \sin (\omega t+\varphi)$ ), and $\left(\sin \alpha \cos \beta_{3} \cos \omega t\right.$, $\left.-\sin \alpha \sin \beta_{3} \sin \omega t\right)$; see Fig. 6. The data show that $\varphi$ is the optical path difference of the two split optical arms from laser source LS to any photodetector. If $\alpha=\beta_{3}=45^{\circ}$, a linearly polarized electric field can be obtained and can be written as $[\cos (\varphi / 2) \cos (\omega t+$ $\varphi / 2), \sin (\varphi / 2) \cos (\omega t+\varphi / 2)]$. The resultant electric field is characterized by a polarization direction swing and angle $\psi=\varphi / 2$ with respect to the $x$ axis; see Fig. 7. Misalignment and manufacture tolerances can usually affect this linearity such that displacement is no longer linear with respect to the phase change. More specifically, if the Lissajous pattern becomes elliptical because of the misalignment and optical component tolerances, the optical signal will encounter a different resolution when it is located to a different part of the ellipse.

\section{A. Azimuth of P1 and QW3}

If azimuth $\alpha$ of polarizer P1 and $\beta_{3}$ of quarter wave plate QW3 are not exactly $45 \mathrm{deg}$, the resultant electric field that enters the photodetectors becomes elliptically polarized. Angle $\psi$ between the long axis of the ellipse and the $x$ axis can be written as

$\tan 2 \psi=\frac{\sin 2 \alpha \sin \varphi}{\sin 2 \alpha \sin 2 \beta_{3} \cos \varphi-\cos 2 \alpha \cos 2 \beta_{3}}$.

It is clear from Eq. (8) that, if $\alpha \neq 45^{\circ}$ and $\beta_{3}=45^{\circ}$, no nonlinear error will be present. However, if $\alpha \neq 45^{\circ}$ and $\beta_{3} \neq 45^{\circ}$, a nonlinear error exists and needs to be addressed.

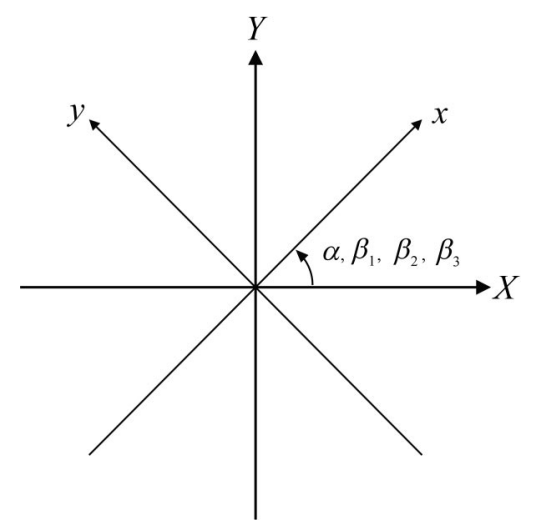

Fig. 5. Coordinate system defined for the optical analysis. $X$ and $Y$ are the global axes where $x$ and $y$ are the fast and slow axes for the quarter wave plate, respectively; $\alpha$ is the polarization transmission angle between $x$ and $X$ for polarizer P1; and $\beta_{1}, \beta_{2}$, and $\beta_{3}$ are the angles between $x$ and $X$ for quarter wave plates QW1, QW2, and QW3, respectively.

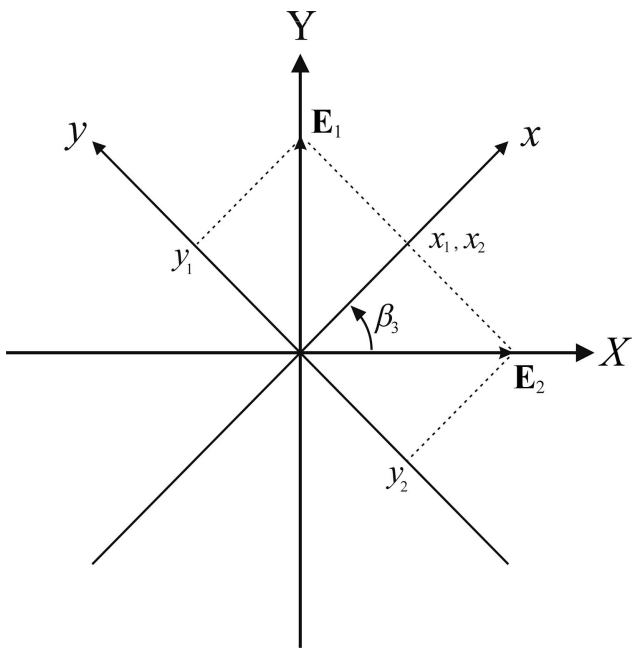

Fig. 6. Electrical field components of the two optical arms projected on a coordinate system.

\section{B. Phase Delay Deviation and Azimuth of QW1}

If there is an extra phase delay $\Delta_{1}$ on azimuth $\beta_{1}$ of quarter wave plate $\mathrm{QW} 1$, the resultant electric field that enters the photodetectors becomes elliptically polarized. Angle $\psi$ between the long axis of the ellipse and the $x$ axis can be shown as

$$
\tan 2 \psi=\tan \beta_{1} \cos 2 \Delta_{1} \tan \varphi
$$

When $\Delta_{1}=0$ and $\beta_{1}=45^{\circ}$, Eq. (9) demonstrates that the resultant electric field beam that enters the photodetectors becomes linearly polarized, i.e., no nonlinear error appears. It is also clear that the phase delay deviation $\Delta_{1}$ is associated with a manufacturing tolerance and certainly cannot be eliminated by alignment.

\section{Phase Delay Deviation and Azimuth of QW2}

Similar to Eq. (9), $\tan 2 \psi=\tan \beta_{2} \cos 2 \Delta_{2} \tan \varphi$ also holds for this case. As corner cube CC used in the

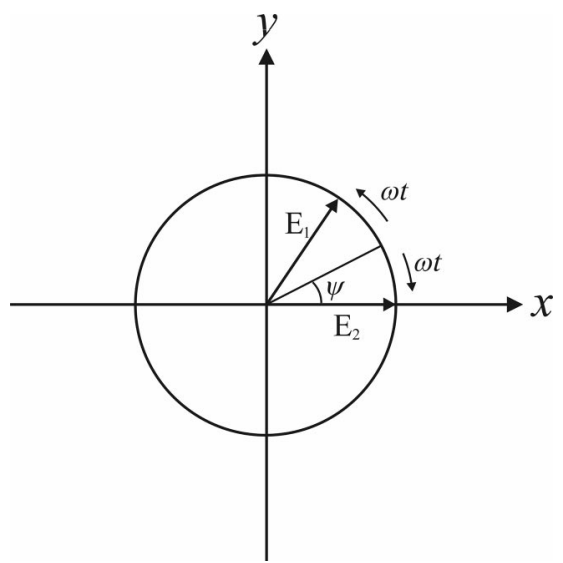

Fig. 7. Optical path differences of two split optical arms from laser source LS to any photodetector under ideal conditions with a linear relationship. 
DiLENS contains a silver coated film on the three reflective surfaces, polarization of the emergent light will show a 180 deg phase shift. This phase shift can be attributed to reflected light beam polarization that undergoes a 180 deg phase shift when the light beam is incident on the silver film at a large incidence angle. However, as the reflectivity of the silver film is approximately $90 \%$, it reduces the amplitude of the emergent light.

\section{Phase Delay Deviation and Azimuth of QW3}

If a phase delay, deviation $\Delta_{3}$, and azimuth $\beta_{3}$ of quarter wave plate QW3 are present, the resultant electric field that enters the photodetectors becomes elliptically polarized. Angle $\psi$ between the long axis of the ellipse and the $x$ axis can be shown as

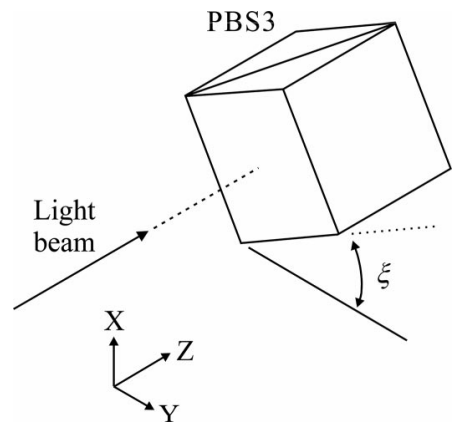

Fig. 8. Polarization misalignment definition of the PBS.

ference beams is formed. Following the above calculation, when $E_{1}{ }^{\prime}$ and $E_{2}{ }^{\prime}$ enter the photodetectors,

$$
\tan 2 \psi=\frac{2\left[\tilde{A} \tilde{D} \sin \left(\Delta_{3}-\varphi\right)-\tilde{B} \tilde{C} \sin \left(\Delta_{3}+\varphi\right)+\tilde{C} \tilde{D} \sin \Delta_{3}-\tilde{A} \tilde{B} \sin \Delta_{3}\right]}{\tilde{B}^{2}+\tilde{D}^{2}-\tilde{A}^{2}-\tilde{C}^{2}-2(\tilde{B} \tilde{D}+\tilde{A} \tilde{C}) \cos \varphi}
$$

where $\tilde{A}=\cos \alpha \sin \beta_{3}, \tilde{B}=\cos \alpha \cos \beta_{3}, \tilde{C}=\sin \alpha$ $\cos \beta_{3}$, and $\tilde{D}=\sin \alpha \sin \beta_{3}$. If $\Delta_{3}=0$ and $\tilde{A} \neq \tilde{B}$ their electric field is elliptically polarized. Angle $\psi^{\prime}$ between the long axis and the $x$ axis becomes

$$
\tan 2 \psi^{\prime}=\frac{2\left[-\tilde{E} \tilde{H} \sin \left(\Delta_{3}-\varphi\right)+\tilde{F} \tilde{G} \sin \left(\Delta_{3}+\varphi\right)+\tilde{E} \tilde{F} \sin \Delta_{3}-\tilde{G} \tilde{H} \sin \Delta_{3}\right]}{\tilde{F}^{2}+\tilde{H}^{2}-\tilde{E}^{2}-\tilde{G}^{2}-2(\tilde{F} \tilde{H}+\tilde{E} \tilde{G}) \cos \varphi}
$$

$\neq \tilde{C} \neq \tilde{D}$, then

$\tan 2 \psi=\frac{-\sin 2 \alpha \sin \varphi}{\left(\cos ^{2} \alpha-\sin \alpha\right) \cos 2 \beta_{3}-\sin 2 \alpha \sin 2 \beta_{3} \cos \varphi}$,

where $\beta_{3}=45^{\circ}$ and $\psi=\varphi / 2$. Therefore, the nonlinear error that is due to factors reflected in Eq. (8) can be eliminated by an optical path adjustment. If $\Delta_{3} \neq 0$ and $\tilde{A}=\tilde{B}=\tilde{C}=\tilde{D}$, Eq. (8) can then be simplified as $\tan 2 \psi=\tan \varphi \cos \Delta_{3}$ which indicates that the nonlinear error caused by phase delay deviation $\Delta_{3}$ of QW3 cannot be eliminated by alignment.

\section{E. Polarization Deviation of PBS1}

From the above analysis it was found that the deviation of $\Delta_{1}, \Delta_{2}, \beta_{1}$, and $\beta_{2}$ results in associated electric components such as $E_{1}{ }^{\prime}$ and $E_{2}{ }^{\prime}$. It can be shown that, if the two beams split by PBS1 are not pure $p$ or $s$ polarization, the same phenomena can be observed. When $E_{1}{ }^{\prime}$ and $E_{2}{ }^{\prime}$ are generated, another set of inter- where $\tilde{E}=p_{1} \cos \beta_{3}, \tilde{F}=p_{1} \sin \beta_{3}, \tilde{G}=p_{2} \sin \beta_{3}$, and $\tilde{H}=p_{2} \cos \beta_{3}$. In addition, $p_{1}$ and $p_{2}$ equal $E_{1}{ }^{\prime} / E_{1}$ and $E_{2}{ }^{\prime} / E_{2}$, respectively. For $\tilde{E}=\tilde{F}=\tilde{G}=\tilde{H}$, we can observe the following relationship:

$$
\tan 2 \psi^{\prime}=-\tan \varphi \cos \Delta_{3} .
$$

\section{Misalignment Effects}

\section{A. PBS3}

Here we discuss the effect of an inaccurate alignment of PBS3, which is the end component of the circular polarization interferometer on the signals. Considering the case of a deviation of the PBS3 polarization $\xi$ (Fig. 8), the error of phase change after the arctangent calculation can be shown as

$$
\varepsilon(\Phi)=\tan ^{-1}\left[\frac{\sin (\Phi+2 \xi)}{\cos \Phi}\right]-\Phi
$$

From Eq. (14) we see that $\varepsilon(\Phi)$ is a function with period $\pi$. At the same time, if the polarization direc- 
tion of PBS3 has a deviation of $\xi$, it may cause signals $P$ and $Q$ to be inclined for $2 \xi$, making the output interference signals appear as an inclined ellipse.

\section{B. Photodetector Misalignment}

If the photodetector is not well aligned, extra phase differences may be observed on the output signals of the photodetector. The amplitudes measured by the four photodetectors and then differentially amplified can be written as

$$
\begin{aligned}
& I_{P}=\tilde{P} \sin \left(4 \Delta \omega t+\tilde{\Phi}_{P}\right), \\
& I_{Q}=\tilde{Q} \cos \left(4 \Delta \omega t+\tilde{\Phi}_{Q}\right),
\end{aligned}
$$

where $\tilde{P}$ represents the swing of signal $P, \tilde{\Phi}_{P}$ is the phase angle of the $P$ signal, $\tilde{Q}$ is the swing of the $Q$ signal, and $\tilde{\Phi}_{Q}$ is the phase angle of the $Q$ signal. From Eqs. (15) and (16) we found that inaccurate alignment of the photodetectors can create extra phase differences on the output interference signals and make them appear as inclined ellipses.

\section{Photodetector Misalignments and PBS3}

If simultaneous inaccurate alignments are found on the photodetectors and PBS3, the deviation of the phase change can be expressed as

$\varepsilon(\Phi)=\tan ^{-1}\left[\frac{\sin \left(\Phi+2 \xi+\Phi_{13}\right)+\sin \left(\Phi+2 \xi+\Phi_{14}\right)}{\cos (\Phi+2 \xi)+\cos \left(\Phi+2 \xi+\Phi_{12}\right)}\right]-\Phi$,

where $\Phi_{12}, \Phi_{13}$, and $\Phi_{14}$ are, respectively, extra phase differences of PD2, PD3, and PD4 that are due to photodetector misalignment with respect to PD1. Furthermore, it can be shown that the interference signals, $I_{P}$ and $I_{Q}$, can be simplified as in Eqs. (15) and (16). It was found that simultaneous inaccurate alignments of the photodetectors and PBS3 can cause the output interference signals to appear as an inclined ellipse. Furthermore, the above analysis shows that the misalignment could generate a nonlinear error.

\section{Encoder Static Misalignment Analysis}

Figure 9(a) shows when the grating is well aligned within the wavefront conjugate optical configuraton, which consists of lens L and mirror M2 placed in specific relative positions as shown in Fig. 3. More specifically, the grating is placed at the front focal point of the lens to form a complete wavefront conjugate optical configuration. The course of a beam through this wavefront conjugate arrangement can be represented by the following matrix based on matrix optics [6]:

$$
\begin{aligned}
& {\left[\begin{array}{cc}
1 & -f \\
0 & 1
\end{array}\right]\left[\begin{array}{cc}
1 & 0 \\
1 / f & 1
\end{array}\right]\left[\begin{array}{cc}
1 & -f \\
0 & 1
\end{array}\right]\left[\begin{array}{cc}
1 & 0 \\
0 & -1
\end{array}\right]\left[\begin{array}{cc}
1 & f \\
0 & 1
\end{array}\right]\left[\begin{array}{cc}
1 & 0 \\
-1 / f & 1
\end{array}\right]} \\
& \times\left[\begin{array}{cc}
1 & f \\
0 & 1
\end{array}\right]\left\{\begin{array}{c}
h \\
\theta
\end{array}\right\}=\left\{\begin{array}{c}
-h \\
\theta
\end{array}\right\} .
\end{aligned}
$$

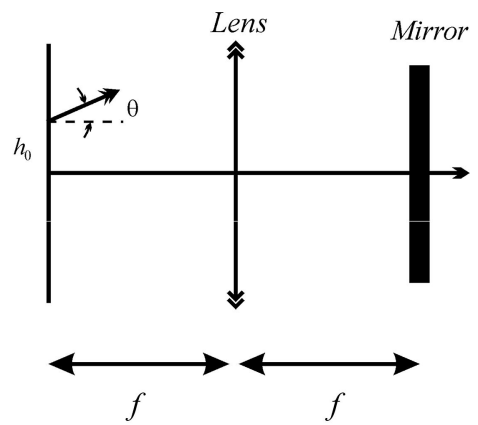

(a)

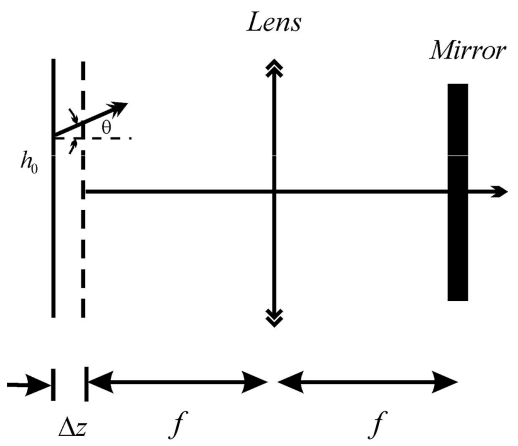

(b)

Fig. 9. Mathematical analysis model for the runout between optical heads and encoder when the optical diffractive location is (a) in focus and (b) out of focus.

Equation (18) clearly demonstrates that the function of the wavefront conjugate optical configuration is to keep the input path of the encoder parallel to the output path. In other words, the structure of the wavefront conjugate optical configuration is capable of keeping the return path of the optical head parallel to the original incident path. Because of these properties, it can be regarded as a virtual corner cube. It can be shown that the wavefront conjugate configuration keeps the incident beam parallel to the output beam despite different head-to-scale static misalignment conditions.

The head-to-scale alignment tolerance can be determined through the encoder static misalignment procedure discussed herein. Figure 10 shows the ex-

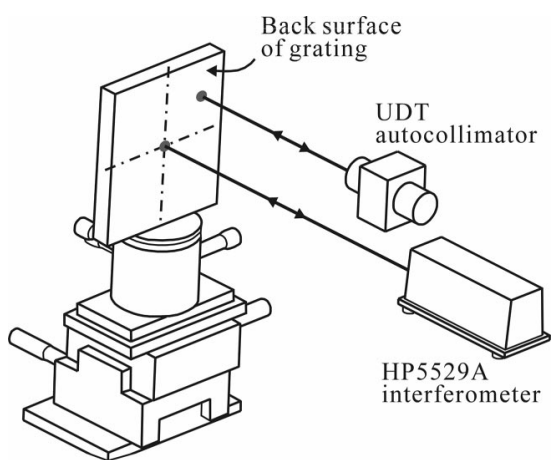

Fig. 10. Experimental setup for determining head-to-scale alignment tolerances. 
Table 1. Comparison of Head-to-Scale Alignment Tolerances of the DiLENS

\begin{tabular}{|c|c|c|c|c|}
\hline $\begin{array}{c}\text { Alignment } \\
\text { Tolerance }\end{array}$ & LightTools & Code V & $\begin{array}{c}\text { Experimental } \\
\text { Results }\end{array}$ & $\begin{array}{c}\text { Traditional } \\
\text { Laser } \\
\text { Encoder }\end{array}$ \\
\hline Roll & \pm 80 arc $\min$ & \pm 78 arc $\min$ & \pm 75 arc $\min$ & \pm 3 arc $\min$ \\
\hline Pitch & \pm 18 arc min & \pm 17 arc min & \pm 15 arc min & \pm 20 arc $\mathrm{min}$ \\
\hline Yaw & $\pm 2.2 \mathrm{deg}$ & $\pm 1.9 \mathrm{deg}$ & $\pm 2 \mathrm{deg}$ & \pm 20 arc $\min$ \\
\hline Stand-off & $\pm 0.51 \mathrm{~mm}$ & $\pm 0.47 \mathrm{~mm}$ & $\pm 0.5 \mathrm{~mm}$ & $\pm 0.2 \mathrm{~mm}$ \\
\hline Offset & $\pm 1.6 \mathrm{~mm}$ & $\pm 1.5 \mathrm{~mm}$ & $\pm 1.5 \mathrm{~mm}$ & $\pm 0.3 \mathrm{~mm}$ \\
\hline
\end{tabular}

perimental setup for the measurement of encoder static misalignment tolerances. An HP5529A laser interferometer, a distance measuring interferometer based on heterodyne interferometry and known to have an approximate $17 \mathrm{~nm}$ distance measurement resolution, was used to perform the feedback control of the head-to-scale distance of the stage driving unit. More specifically, the distance measured was the same regardless of whether we measured the roll, pitch, or yaw tolerances. Our experimental setup ensured that the standoff effect did not involve the roll, pitch, or yaw tolerances. The United Detector Technology (UDT) autocollimator was used to determine the static angular misalignment (roll, pitch, and yaw). When the interference signal was not received by the photodetectors, it indicated that the runout of the encoder exceeded the allowable range of the optical heads. Table 1 details the results of the experimental setup and compares the tolerance values as calculated by LightTools and Code V software simulation programs and by our experimental measurements. The data show that the introduction of the wavefront conjugate configuration into our newly developed DiLENS results in an alignment tolerance between the optical heads and the optical encoder that is 5 to 25 times higher than what is found with a traditional laser encoder [8].

\section{Experimental Verification}

Performance of the HP5529A laser interferometer was compared with our novel DiLENS to evaluate the measurement accuracy of the latter. Figure 11 shows the experimental structure for verification. Sending the signal output from each pair of photo-

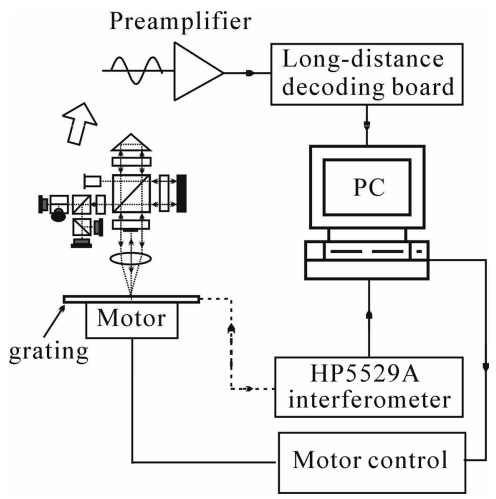

Fig. 11. Experimental configuration for verification of the DiLENS.

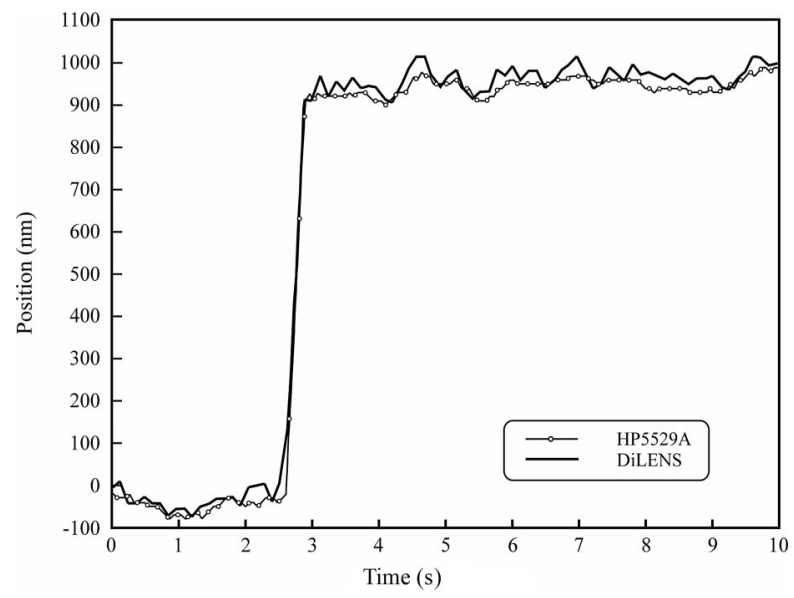

Fig. 12. Comparative verification of the DiLENS and the HP5529A when the encoder platform moves $1 \mu \mathrm{m}$.

diodes, i.e., the PD1 and PD2 pair and the PD3 and PD4 pair, into a differential amplifier to remove the common mode DC signal and implementing an error-corrected phase method provided us with a way to eliminate completely the errors associated with a case when the Lissajous pattern becomes elliptical [9]. More specifically, the interference signal received by the photodetector pairs of the circular polarization interferometer was amplified differentially before being digitized by a high-speed analog-to-digital converter. Subsequently, the signal was fed into a field-programmable gate array (FPGA), produced by AHEAD Optoelectronics, Taiwan, and developed by the Nano-Bio-MEMS Laboratory of the National Taiwan University, for encoder displacement by use of an arctangent calculation [9]. The result of the FPGA was then transferred back to the computer by a digital input-output card. Finally, LabVIEW software was used to display the displacement of the grating. The encoder displacement was independently measured with an HP5529A interferometer, a commonly used laboratory instrument to measure displacements. Figures 12 to 14 detail the comparative veri-

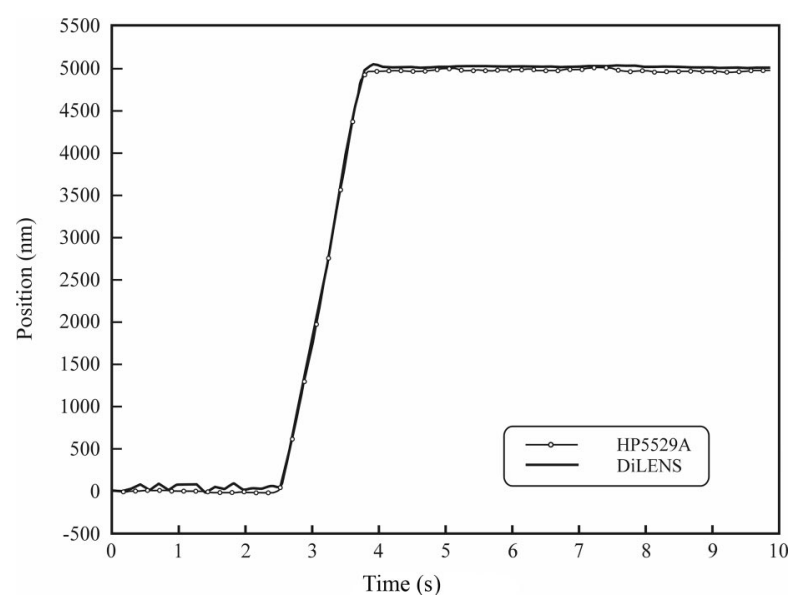

Fig. 13. Comparative verification of the DiLENS and the HP5529A when the displacement of the encoder platform is $5 \mu \mathrm{m}$. 

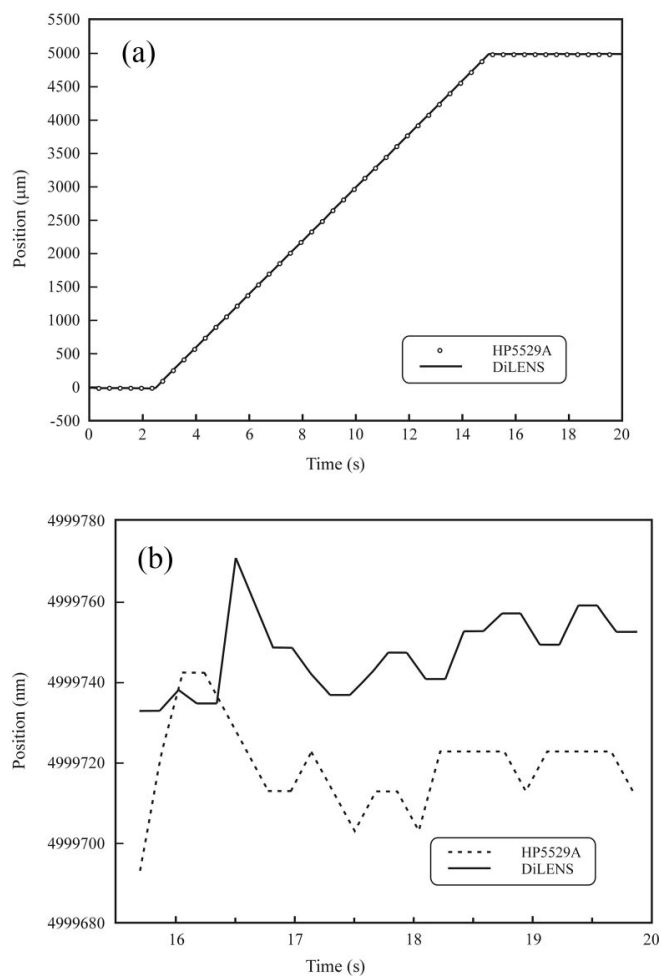

Fig. 14. Verification of the DiLENS and the HP5529A when the displacement of the encoder platform is $5 \mathrm{~mm}$ at (a) a full path and (b) enlarged verification between 15.5 and $20 \mathrm{~s}$.

fication of the DiLENS and the HP5529A under a different displacement of the encoder system $(\Delta X$ $=1,5$ and at $5000 \mu \mathrm{m}$ ). The solid curve represents the determined displacement on the DiLENS and the dotted curve represents the determined displacement on the HP5529A. From the experimental results we found that the locating error between the displacement of the DiLENS and the HP5529A was less than $37.33 \mathrm{~nm}$, with a standard deviation of $25.4 \mathrm{~nm}$.

\section{Conclusions}

We conducted detailed experiments to verify the theoretical predictions and the overall system specifications of our newly developed DiLENS. The calibration instrument used was a HP5529A laser displacement interferometer. Even when running the calibration under normal laboratory conditions, i.e., without extensive environmental control, the displacement discrepancy between the calibration instrument and our newly developed DiLENS was a mere $37.3 \mathrm{~nm}$, with a $25.4 \mathrm{~nm}$ standard deviation.

In summary, we developed a novel high resolution linear encoder that has potential for wide applicability because of its high manufacturing tolerance and less sensitivity to external environmental conditions. Our newly developed encoder has many advantages when compared with traditional laser encoders, such as the capability of achieving measurement resolutions in nanometers.

This research was performed at the NanoBioMEMS Laboratory at the Institute of Applied Mechanics of National Taiwan University. This project was partially supported by the Taiwan Ministry of Economic Affairs (MOEA), Department of Industrial Technology Development Program for Academia, 91EC-17-A-05-S1-0017, and partially supported by the National Science Council under project NSC92-2622E-002-006. Special thanks to Optical Research Associates for providing us with the optical simulation program.

\section{References}

1. N. Bobroff, "Recent advances in displacement measuring interferometry," Meas. Sci. Technol. 4, 907-926 (1993).

2. S. Hosoe, "Highly precise and stable laser displacement measurement interferometer with differential optical passes in practical use," Nanotechnology 4, 81-85 (1993).

3. V. G. Badami and S. R. Patterson, "A frequency domain method for the measurement of nonlinearity in heterodyne interferometry," Precis. Eng. 24, 41-49 (2000).

4. D. Lin, H. Jiang, and C. Yin, "Analysis of nonlinearity in a high-resolution grating interferometer," Opt. Laser Technol. 32, 95-99 (2000).

5. K. G. Masreliez, "Position detection and method of measuring position," U.S. patent 5,104,225 (14 April 1992).

6. F. J. Pedrotti and L. S. Pedrotti, Introduction to Optics (Prentice-Hall, 1990).

7. GSolver User Manual, http://www.gsolver.com.

8. S. Ichikawa and H. O. Kawasaki, "Diffraction-type optical encoder with improved detection signal insensitivity to optical grating gap variations," U.S. patent 4,943,716 (24 July 1990).

9. W.-J. Wu, C.-K. Lee, and C.-T. Hsieh, "Signal processing algorithms for Doppler effect based nanometer positioning systems,” Jpn. J. Appl. Phys. Part 1 38, 1725-1729 (1999). 